\title{
Thermoconvective instabilities of nematic liquid layers : new theoretical predictions
}

\author{
M. G. Velarde $\left(^{+}\right)$and I. Zúñiga \\ Departamento de Física de Fluidos, C-3, Universidad Autónoma de Madrid, Cantoblanco (Madrid), Spain
}

(Reçu le 13 juin 1978, révisé les 7 novembre 1978 et 27 février 1979, accepté le 7 mars 1979)

\begin{abstract}
Résumé. - Nous présentons l'étude systématique de la stabilité locale (linéaire) d'une couche nématique chauffée par le bas ou par le haut. Des résultats nouveaux sont rapportés touchant les modes stationnaires et surstables qui peuvent se produire dans le cas d'instabilités dues à la poussée d'Archimède en présence ou non d'un champ magnétique externe pris parallèle au directeur du nématique. Le cas d'une instabilité due à des inhomogénéités de tension superficielle est aussi considéré. La présente note est une étude complémentaire et plus systématique des travaux antérieurs de Guyon et collaborateurs et de Lekkerkerker et donne une vue unifiée et globale de l'éventail des phénomènes dont une couche de nématique peut être le siège lorsqu'on la chauffe par le haut ou par le bas.
\end{abstract}

\begin{abstract}
A systematic study of local stability (linear analysis) of nematic liquid layers heated from below or above is presented. New theoretical findings about steady and overstable modes of convection are discussed for the cases of buoyancy-driven convection in the presence or not of a magnetic field parallel to the director. The case of surface tension-driven convection is also presented. This note completes some earlier work by Guyon and collaborators, and Lekkerkerker, and constitutes a unified approach to the rather disparate phenomena that appear in the general Rayleigh-Bénard problem for nematic layers.
\end{abstract}

1. Introduction. - During the past few years there has been considerable interest in the stability analysis of nematic liquid layers heated from below or above (Rayleigh-Bénard problem) though none of the existing studies [1-5] have yet reached the level of completeness so far in the standard case of isotropic liquids [6]. This is partially due to the additional complexity brought into the hydrodynamics by the anisotropic properties of the nematic liquid.

In planar and homeotropic layers heated from below or above steady transitions to convection were the concern of Dubois-Violette [1] and Barrat and Sloan [3] (see also [2] for a pioneering though less complete study). Neither overstable modes of instability nor the possibility of steady transitions in planar layers heated from above were discussed by these authors. The present note extends their results with a discussion of overstability and the delineation of regions where a steady transition is indeed possible in planar layers heated from above.

$\left(^{+}\right)$Also at Laboratoire de Dynamique et Thermophysique des Fluides, Université de Provence, Centre de Saint-Jérôme, 13397 Marseille, Cedex 4, France.
The discovery of overstable modes of convection is due to Lekkerkerker [4]. However, his short letter cannot be considered a systematic analysis nor does it provide a quantitative estimate of the frequency of oscillation. Guided by Lekkerkerker's finding we have made a complete study of this linear mode of instability in homeotropic layers heated from below. Lekkerkerker's analysis bears a number of points in common with the Soret-driven instability discussed by earlier authors (see for instance [7]). His study had the merit of attracting the attention of experimentalists to an interesting phenomenon whose industrial relevance might very well soon appear.

Experiments concerning the onset of convection in homeotropic layers heated from below have been recently conducted by Guyon, Pieranski and Salan as an extension of earlier work on convective instability in nematics [8-12]. They have confirmed the basic finding of ref. [4] and have also studied the dependence of the linear frequency of oscillation with a magnetic field aligned parallel to the director. It is indeed remarkable that a simple three-mode analysis of the stability problem discussed by Guyon, Pieranski and Salan already gives quantitative estimates in fairly good agreement with their experimental 
findings [12]. In the present note we establish their results in a more systematic manner, and we discuss also some different geometries for the boundary conditions.

Lastly, in a recent note, Guyon and Velarde [5] have given a one dimensional two-mode analysis of the stability of a planar nematic layer heated from below and open to the ambient air. Here the action of surface tension stresses at the open interface couples to body forces to drive the system convectively unstable. As already indicated in ref. [5] the present note aims at further completing the picture sketched by Guyon and Velarde.

Section 2 deals with a short description of the linear stability problem, and the boundary conditions to be considered. The Galerkin method [13] is used to get qualitative and quantitative results that are presented in section 3. Comparison with presently available experimental data is also discussed in section 3. After completion of this work we have learned of a related though rather different approach to the same problems (Lekkerkerker, private communication) where some of the results reported here are obtained.

2. Evolution of infinitesimal disturbances. - We shall restrict consideration here to sufficient conditions for instability of a motionless horizontal nematic liquid layer heated from below or above. Thus, in this section we present equations for the evolution of disturbances in the velocity, temperature, and director fields. As a matter of fact we shall limit ourselves to the analysis of two dimensional disturbances only. For the planar configuration the director is $(1,0,0)$ and the corresponding disturbances are $n=(0,0, n)$ and $v=(u, 0, w)$ for the director and the velocity fields respectively. For the homeotropic configuration with director $(0,0,1)$ the corresponding disturbances to be considered are $n=(n, 0,0)$ and $v=(u, 0, w)$ respectively. In both geometries $\theta$ will denote temperature disturbances from the initially linear, steady state. We shall follow the notation and approach developed earlier by various authors $[1,3,14,15]$.

2.1 Planar configuration. - A dimensionalization of the dimensional equations (see for instance [14]) is here achieved by using the following scales

$$
\begin{array}{r}
\text { \{time : } \tau=\left(\lambda_{1}+\lambda_{2}\right) d^{2} k_{3}^{-1}, \text { length }: L=d, \\
\text { temperature : } \left.\theta=\beta d k_{a}\left(k_{\|}+k_{a}\right)^{-1}\right\}
\end{array}
$$

where $\lambda_{1}=\alpha_{2}-\alpha_{3} ; \lambda_{2}=\alpha_{5}-\alpha_{6}=-\left(\alpha_{2}+\alpha_{3}\right)$, and $k_{a}=k_{\|}-k_{\perp} \cdot \beta$ is the thermal gradient across the layer of thickness, $d$.

Following the notation used in [14] $\alpha_{2}, \alpha_{3}, \alpha_{5}$ and $\alpha_{6}$ are viscosity coefficients; $k_{\|}$and $k_{\perp}$ are thermal conductivities, respectively parallel and orthogonal to the director ; $k_{1}$ and $k_{3}$ are Frank's constants.
With $w, \theta$ and $n$ denoting respectively vertical velocity, temperature and director disturbances from the motionless steady state, the following equations hold

$$
\begin{aligned}
& \frac{2 \rho k_{1} \sigma}{\eta_{a}\left(\lambda_{1}+\lambda_{2}\right)}\left(D^{2}-a^{2}\right) w+\frac{2 i \alpha_{3} a \sigma}{\eta_{a}}\left(D^{2}+\lambda a^{2}\right) n= \\
& =R a^{2} \theta+\left(D^{4}-\eta a^{2} D^{2}+\eta_{1} a^{4}\right) w \\
& \frac{-i a \lambda_{1} \sigma n}{\left(\lambda_{1}+\lambda_{2}\right)}=i a\left(D^{2}-k a^{2}\right) n-\frac{1}{2}\left(D^{2}+\lambda a^{2}\right) w \\
& \frac{k_{1} \sigma \theta}{k_{\|}\left(\lambda_{1}+\lambda_{2}\right)}=\left(D^{2}-x a^{2}\right) \theta+i a n+\frac{k_{1} w}{k_{a}\left(\lambda_{1}+\lambda_{2}\right)}
\end{aligned}
$$

where

$$
\begin{gathered}
\lambda=\left(\lambda_{2}-\lambda_{1}\right)\left(\lambda_{1}+\lambda_{2}\right)^{-1}=\alpha_{2} \alpha_{3}^{-1} \\
x=\left(k_{\|}+k_{a}\right) k_{\|}^{-1}, \quad k=k_{3} k_{1}^{-1}+\chi_{a} H^{2} d^{2} k_{1}^{-1} a^{-2} \\
\eta_{a}=\alpha_{4}+\alpha_{3}+\alpha_{6}, \quad \eta_{b}=\alpha_{4}+\alpha_{5}-\alpha_{6} \\
\text { and } \quad \eta_{a}=\frac{\left(2 \alpha_{1}+\eta_{a}+\eta_{b}\right)}{\eta_{a}}, \quad \eta_{1}=\frac{n_{b}}{\eta_{a}} \\
R=-4 \rho g \alpha k_{a} \beta d^{4} \alpha_{3} k_{\|}^{-1} k_{1}^{-1} \eta_{a}^{-1}
\end{gathered}
$$

is the (planar) Rayleigh number. $\rho$ denotes the liquid density, $H$ is the field strength, and the remaining symbols have their definition in ref. [14]. $D$ accounts for $d / \mathrm{d} Z$ (derivative along the vertical coordinate $Z$ ). $a$ is a dimensionless horizontal wavenumber and $\sigma$ is the time constant that defines stability $(\operatorname{Re} \sigma>0$, unstable; $\operatorname{Re} \sigma<0$, stable).

2.2 Homeotropic CONFIGURATION. - A dimensionalization is now performed using the following scales :

\{ time : $\tau=\left(\lambda_{2}-\lambda_{1}\right) d^{2} k_{3}^{-1}$, length $: L=d$

and temperature : $\left.\theta=\beta d k_{a}\left(k_{\|}+k_{a}\right)^{-1}\right\}$.

Then the following equations hold

$$
\begin{gathered}
\frac{2 \rho k_{3} \sigma}{\eta_{b}\left(\lambda_{2}-\lambda_{1}\right)}\left(D^{2}-a^{2}\right) w+\frac{2 i a \alpha_{2} \sigma}{\eta_{b}}\left(D^{2}+\lambda^{\prime} a^{2}\right) n= \\
=R a^{2} \theta+\left(D^{4}-\eta^{\prime} a^{2} D^{2}+\eta_{1}^{\prime} a^{4}\right) w \\
\frac{i a \lambda_{1} \sigma n}{\left(\lambda_{1}-\lambda_{2}\right)}=i a\left(D^{2}-k^{\prime} a^{2}\right) n-\frac{1}{2}\left(D^{2}+\lambda^{\prime} a^{2}\right) w \\
\frac{\sigma k_{3} \theta}{\left(k_{\|}+k_{a}\right)\left(\lambda_{2}-\lambda_{1}\right)}=\left(D^{2}-x^{\prime} a^{2}\right) \theta+ \\
\quad+i a n+\frac{k_{3} w}{k_{a}\left(\lambda_{2}-\lambda_{1}\right)}
\end{gathered}
$$

where for convenience

$$
\begin{gathered}
\lambda^{\prime}=\lambda^{-1}, \quad x^{\prime}=x^{-1}, \quad k^{\prime}=k_{1} k_{3}^{-1}+\chi_{a} H^{2} d^{2} k_{3}^{-1} a^{-2}, \\
\eta^{\prime}=\eta / \eta_{1}, \quad \eta_{1}^{\prime}=\eta_{1}^{-1} .
\end{gathered}
$$


The (homeotropic) Rayleigh number is

$$
R=-4 \rho g \alpha k_{a} \alpha_{2} \beta d^{4}\left(k_{\|}+k_{a}\right)^{-1} k_{3}^{-1} \eta_{b}^{-1} .
$$

To the evolution equations given above we now add the boundary conditions (b.c.). Various types are considered here :

(a) Mechanical b.c.

a.1) Rigid plates : $w=D w=0$.

a.2) Stress-free interfaces : $w=D^{2} w=0$.

a.3) Surface tension-driven stresses : $w=0$ and $D^{2} w=-a^{2} M \theta$ in which $M$ accounts for a parameter (Marangoni number) to be defined below. It is to be considered here as an additional eigenvalue parameter.

(b) Thermal b.c.

b. 1) Conducting plates : $\theta=0$.

b.2) Insulating boundaries : $D \theta=0$.

3. Numerical results and comparison with experiment. - All quantitative results reported in this paper refer for convenience to MBBA layers. We shall consider separately the planar and homeotropic configurations in the absence of a magnetic field and discuss subsequently the additional influence of the field (parallel to the director).

Our findings give the results obtained by Guyon, Pieranski and Salan [12] using a simplified model. Lastly, surface tension effects will be considered and

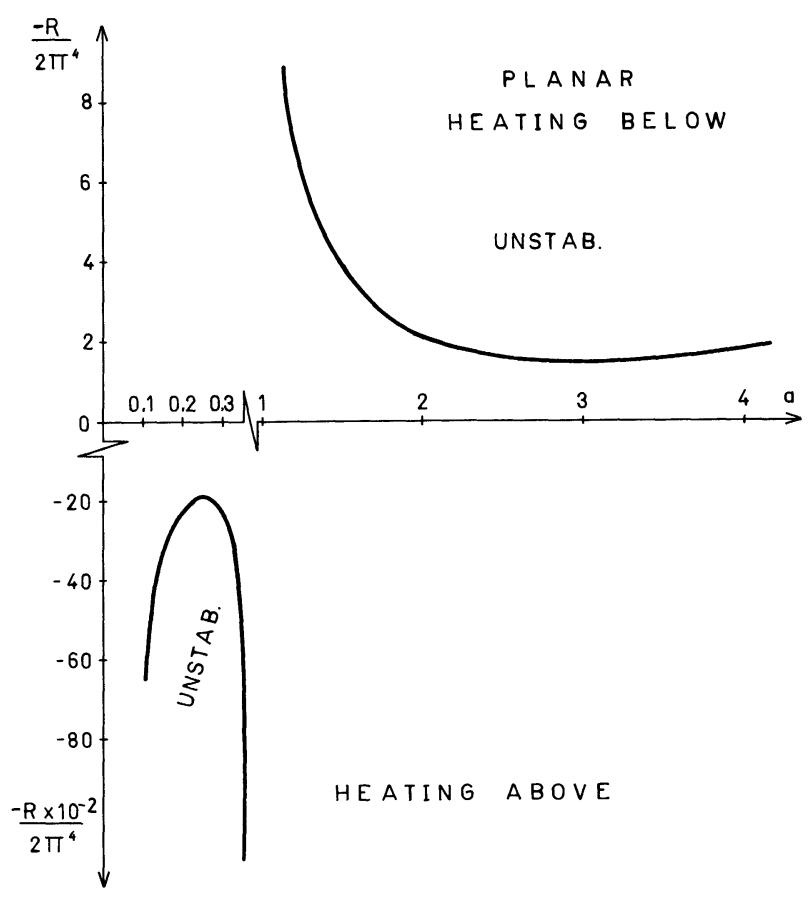

Fig. 1. - Neutral stability curves for steady transitions in planar configurations. The upper and lower portions have a common vertical asymptote. There is no appreciable difference between our results and the predictions given in refs. $[1,3]$ for the neutral curve when heating from below. the results reported here complete an earlier note by Guyon and Velarde [5].

3.1 Planar configuration. - Figure 1 depicts the neutral stability curves found for both ways of heating (below and above). Neutral steady transitions are found and no overstability appears in this case.

Using the Galerkin method to a first-order approximation, with trial functions :

$$
w=(1-Z)^{2} Z^{2}, \quad n=\theta=Z(1-Z),
$$

which satisfy the boundary conditions :

$$
w=D w=n=\theta=0 \quad \text { at } \quad Z=0 \quad \text { and } \quad Z=1,
$$

we get the following critical parameters $\left({ }^{1}\right)$ :

a) Heating from below : $R_{\mathrm{c}}=-291.9, a_{\mathrm{c}}=3.0$. For a layer thickness of $d=5 \mathrm{~mm}$ the corresponding temperature difference is $\Delta T_{\mathrm{c}}=-0.022^{\circ} \mathrm{C}$. These results fit nicely with the predictions of DuboisViolette [1] and Barrat and Sloan [3], and with the experimental data [8].

b) Heating from above: $R_{\mathrm{c}}=0.389 \times 10^{6}, a_{\mathrm{c}}=0.28$. Thus the critical temperature difference is $\Delta T_{\mathrm{c}}=29^{\circ} \mathrm{C}$ for $d=5 \mathrm{~mm}$. Clearly, this instability when heating from above could only be of relevance for relatively thick layers. Its operation eventually demands that

$$
\left|\alpha_{3} \partial u / \partial Z\right|>\left|\alpha_{2} \partial w / \partial X\right|
$$

for which a plausible justification is given below. As $\alpha_{2}$ is about a hundred times $\alpha_{3}$, at least for almost circular rolls where $\partial u / \partial Z \sim \partial w / \partial X$ we generally have

$$
\left|\alpha_{3} \partial u / \partial Z\right|<\left|\alpha_{2} \partial w / \partial X\right|
$$

in a planar configuration. However, with highly elongated cells $\left(a_{\mathrm{c}} \ll 1\right), u$ varies more abruptly than $w$ in the corresponding variable, and overturning in the mode of operation of the viscous torques is

( $\left.{ }^{1}\right)$ In the Galerkin method one searches for an approximate solution of the differential problem by imposing the orthogonality of the trial functions to each of the elements of the set used to construct these trial functions, as described, for instance, in ref. [13] Such set is assumed to be complete in the corresponding space of solutions. The requirement that the set be complete is. a matter of mathematical necessity for the theory (convergence properties of the approximation scheme, etc.). In practice, however, one ignores this and merely retains the first few elements in the expansions of the trial functions. Low order approximations may show a strong dependence on the choice of the trial functions though experience tells [13] that already first-order truncations have proven to be very useful and rather accurate. Calculations to the second order involve already extremely cumbersome algebra. We have neglected them here, and have checked that first-order approximations compare quite well with results already known. The upper portion of figure 1 corresponds to the results found by Dubois-Violette [1] and Barrat and Sloan [3]. They follow the curve that we have obtained using a first-order approximation. 
possible provided $\partial u / \partial Z$ is about a hundred times larger than $\partial w / \partial X$. Some confidence in this statement can be obtained if certain results known in the isotropic case are assumed to be valid for the nematic layers.

With $a_{\mathrm{c}} \sim \pi\left(\lambda_{\mathrm{c}} \sim 2 d\right)$ we have an almost circular roll. Here $l=\lambda / 4=d / 2$ is the distance between a maximum of $w$ (termed $w_{\mathrm{m}}$ ) and its vanishing value. Thus, over a cell we have to a first approximation

$$
\partial w / \partial X \sim w_{\mathrm{m}} / l .
$$

For the horizontal component of the velocity, the maximum value is located somewhere in the middle of the upper or lower portions of the cell gap. Thus the distance between the maximum and the vanishing value is about $l^{\prime}=d / 4$. Over a cell we have to a first approximation $\partial u / \partial Z \sim u_{\mathrm{m}} / l^{\prime}$. Assuming that $w_{\mathrm{m}} \sim u_{\mathrm{m}}$ comparison of the two velocity gradients yields a ratio of about two : $\partial u / \partial Z \sim 2 \partial w / \partial X$ which is a rough but valid estimate. Consider now the case of an elongated cell, for which $a_{\mathrm{c}} \sim \pi / 10\left(\lambda_{\mathrm{c}} \sim 20 d\right)$. In this case, comparison of the two velocity gradients yields $\partial u / \partial Z \sim 20 \partial w / \partial X$ which is already an order of magnitude higher than in the circular roll. From the estimates made, the following conclusions can be reached concerning the operation of the viscous torques :

(i) Circular roll : $\left|\alpha_{2} \partial w / \partial X\right|$ is about fifty times

$$
\left|\alpha_{3} \partial u / \partial Z\right|
$$

(ii) Elongated cell : $\left|\alpha_{2} \partial w / \partial X\right|$ is about five times $\left|\alpha_{3} \partial u / \partial Z\right|$.

The operation of the viscous torques in the circular cell case hinders convective motions whereas in the case of elongated rolls its influence is less sensitive, at least by an order of magnitude. This argument merely gives plausibility to the eventually abnormal operation of the torques. A more complete argument and a serious quantitative support to such an abnormal mode of operation of the viscous torques can only be obtained by computing the non linear flows, a problem which is admittedly beyond the modest scope of the presen' note.

3.2 Homeotropic CONFIGURATION. - The neutral stability curves are given on figure 2 . The results have been obtained using the same trial functions and b.c. as in the planar configuration. Steady transition has been found when heating from above. For the case of conducting boundaries the critical values are :

$$
R_{\mathrm{c}}=4547, a_{\mathrm{c}}=2.85,
$$

which for a one-millimeter thick layer corresponds to $\Delta T_{\mathrm{c}}=5.37^{\circ} \mathrm{C}$, which compares rather well with the predicted value of $5^{\circ} \mathrm{C}$ by Dubois-Violette [1] and the reported experimental finding of $5.6^{\circ} \mathrm{C}$ [9]. For stress-free, and good conducting boundaries we

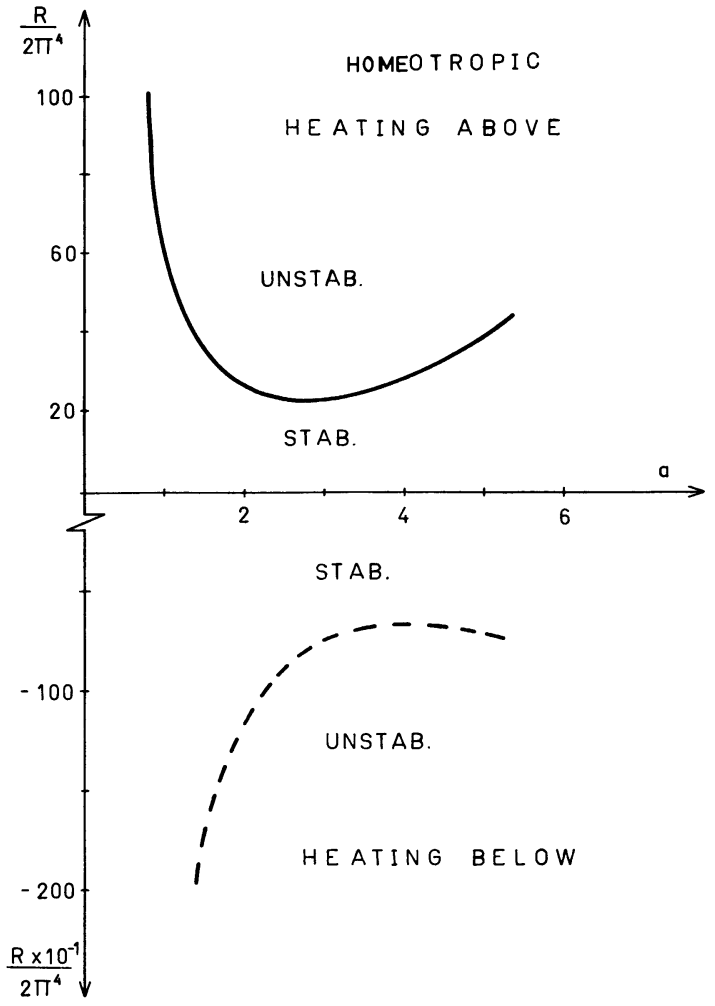

Fig. 2. - Neutral stability curves for steady transitions (upper part) and overstability (dotted line) in homeotropic layers.

have found $R_{\mathrm{c}}=1252, a_{\mathrm{c}}=2.25$, which for $d=1 \mathrm{~mm}$ yields $\Delta T_{\mathrm{c}}=1.5^{\circ} \mathrm{C}$.

Overstable modes of convection have also been located when heating the layer from below as already predicted by Lekkerkerker [4]. The critical values are : $R_{\mathrm{c}}=-0.13 \times 10^{7}, a_{\mathrm{c}}=4$, which for $d=5 \mathrm{~mm}$ yields $\Delta T_{\mathrm{c}}=-12^{\circ} \mathrm{C}$ (heating from below). The associated linear period of oscillation is about 120 seconds, which fits fairly well with recent findings by Guyon, Pieranski and Salan $[11,12]$.

3.3 EFFeCT OF MAGNeTIC FIELD. - 3.3.1 Homeotropic configuration. - Guyon and collaborators $[8,9]$ have already reported on the role of a magnetic field upon the onset of convection in a nematic liquid layer heated from below or above, and some theoretical results also exist in the literature [3]. These authors have found the following empirical law

$$
\Delta T_{\mathrm{c}}(H)=\Delta T_{\mathrm{c}}(H=0)\left[1+\left(H / H_{0}\right)^{2}\right]
$$

assumed to be valid for small enough values of the field strength. Here $H_{0}$ is about $20 \mathrm{G}$ at five millimeter thickness. Their law is valid for both the planar and homeotropic cases provided the magnetic field is parallel to the director. A three-mode theory has also been given by Guyon, Pieranski and Salan. To further complete their simplified picture, which gives, however, a nice explanation of their data and as a natural outcome of our analysis we have also studied the influence of the magnetic field upon the onset of 
Bénard convection in the layer. The predicted results are reported in figure 3. Agreement between our theory and the recent experimental data by Guyon, Pieranski and Salan [10-12] is remarkable up to about $120 \mathrm{G}$. Beyond this value our theory predicts that the relation between the thermal gradient and the field strength should find an asymptote at about $300 \mathrm{G}$, as indicated in figure 3 . Note that all these quantitative results refer to a $d=5 \mathrm{~mm}$ layer thickness.

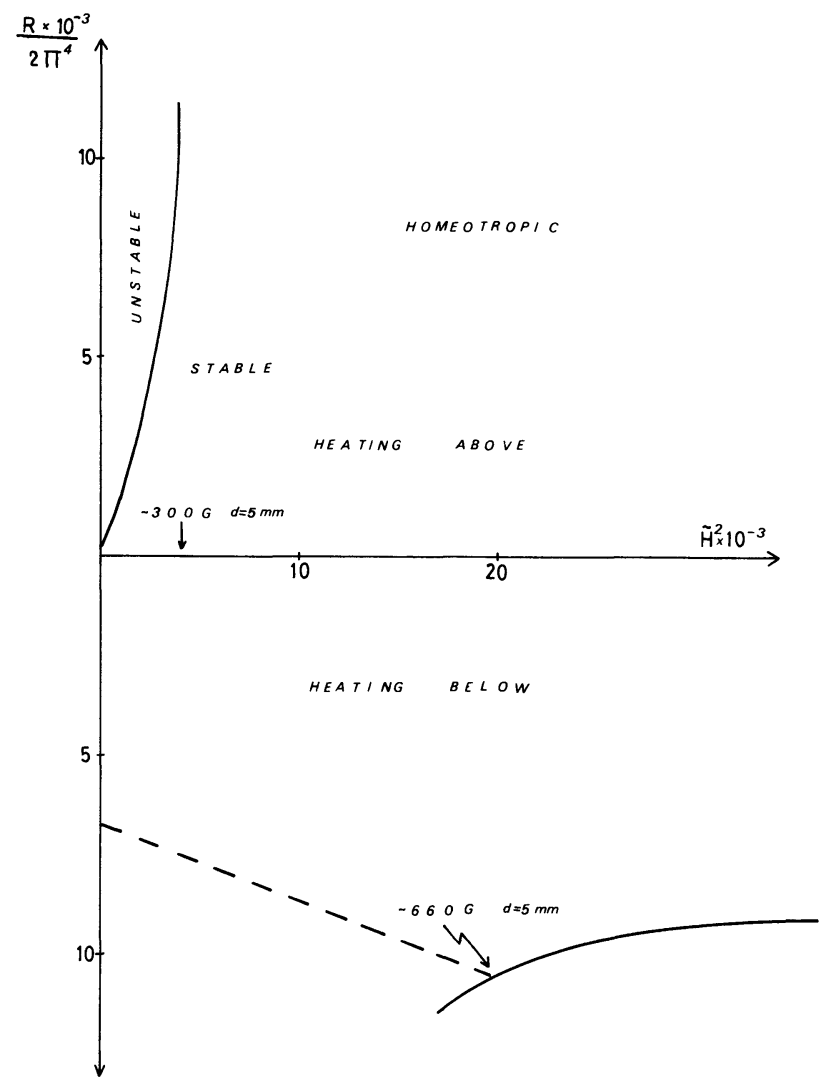

Fig. 3. - Neutral stability curves for a homeotropic configuration in the presence of a magnetic field parallel to the director. Heavy and dotted lines correspond respectively to steady and overstable modes of instability. In the absence of magnetic field the ordinate is 4 547. Large values of the field (at and above $660 \mathrm{G}$ for $d=5 \mathrm{~mm}$ ) inhibit the oscillations. The abscissa refers to dimensionless quantity

$$
\tilde{H}^{2} \equiv \chi_{a} d^{2} H^{2} / k_{3}
$$

Overstable modes in homeotropic configuration are described in the lower portion of figure 3 . The dotted line provides the critical Rayleigh number for overstability as a function of the applied magnetic field. According to an argument already developed in ref. [4] the overstable mode originates in the fact that the relaxation time of director fluctuations is larger than the thermal relaxation time. With the increasing values of the magnetic field these two relaxation times approach each other in magnitude. They have a common value at about $660 \mathrm{G}$, at which point overstability yields to steady convection.
The heavy line in the lower portion of figure 3 corresponds to neutral stable states and critical Rayleigh numbers at given magnetic fields. Below $660 \mathrm{G}$ there is nothing of relevance as the system is already oscillatory unstable at lower values of the Rayleigh number. It should be noted that in this portion of figure 3 the magnetic field has a slightly destabilizing effect. As already pointed out in ref. [9] in a homeotropic layer heated from below the thermal focalization associated with change in orientation of the director rather stabilizes the system. This is due to the role played by the dominant viscous torque. Thus the magnetic field tends to suppress any change in orientation of the director as it forces the molecules to align in a direction orthogonal to the horizontal plates, which inhibits the stabilizing effect of the viscous torque. On the other hand, we have found that as should be expected, for strong enough magnetic fields the critical Rayleigh number becomes field-independent. The critical Rayleigh number approaches the value associated with the isotropic fluid layer, which corresponds to about $\Delta T_{\mathrm{c}} \sim 13^{\circ} \mathrm{C}$ for a $d=5 \mathrm{~mm}$ thickness.

Figure 4 depicts the values of the frequency of oscillation with respect to the square of the field strength. Again this law nicely fits recent findings by Guyon and collaborators. In particular, the transition oscillatory-steady convection found at about $660 \mathrm{G}$ agrees well with their data [12].

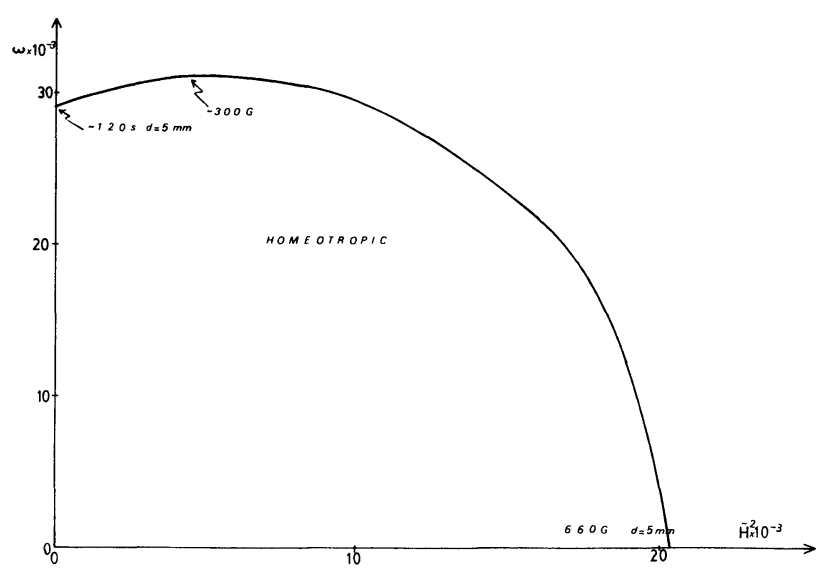

Fig. 4. - Dimensionless frequency of oscillation for linearly overstable modes of instability, as a function of the applied field in homeotropic layers. Large values of the field tend to inhibit the fluctuations of the director, thus reducing the problem to the isotropic case. The period of 120 seconds corresponds to a layer thickness of $d=5 \mathrm{~mm}$. Proper dimensionalization of $T=2 \pi / \omega$ is obtained by using the scaling factor $\left(\lambda_{2}-\lambda_{1}\right) d^{2} / k_{3}$. The quantity $\tilde{H}^{2}$ is as in the preceding figure 3 .

3.3.2 Planar configuration. - The magnetic field (parallel to the director) plays a stabilizing role. The instability when heating from above, present in zero field, tends to be suppressed by the field. For the instability when heating from below (see figure 5) 


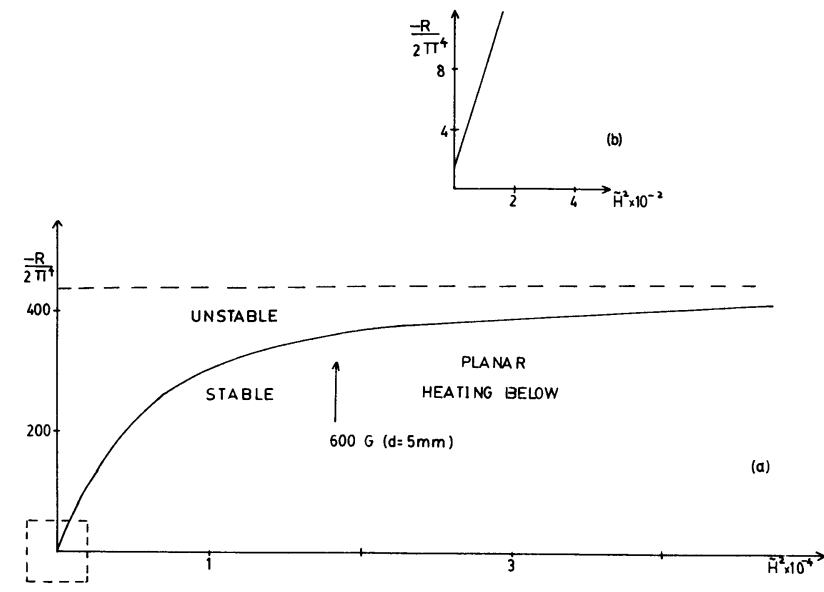

Fig. 5. - (a) Neutral stability curves for the onset of steady convection in a planar layer subjected to a thermal gradient and a magnetic field parallel to the director. The abscissa refers to the dimensionless quantity

$$
\tilde{H}^{2} \equiv \chi_{a} d^{2} H^{2} / k_{1} .
$$

(b) Enlarged view of the region near the origin in part (a) which corresponds to low values of the applied field.

there is an increase of the critical Rayleigh number with the field until a constant value of the thermal gradient is attained (for a $d=5 \mathrm{~mm}$ thickness this comes to about $600 \mathrm{G}$ ). It also appears here that the critical gradient is two or three times smaller than the corresponding values for isotropic liquids.

3.4 SURFACE TENSION-DRIVEN INSTABILITY. - The following trial functions have been used :

$$
w=Z^{2}(1-Z), \quad n=Z(1-Z),
$$

and $\theta=Z(1-Z / 2)$. Then at $Z=0$ we have the b.c. a.1) and b.1) satisfied whereas at the open interface $(Z=1)$ considered insulating b.2) and obeying a.3), $w$ does not satisfy the b.c. and a residual is generated. This additional residual brings a supplementary equation and is treated in the standard manner described, for instance, in ref. [13].

3.4.1 Planar configuration. - A dimensionalization of the b.c. yields

$$
D^{2} w=-2 a^{2} \sigma_{0} k_{a} d^{2} \alpha_{3} \beta \theta / k_{\|} \rho v k_{1}
$$

and by analogy with the isotropic case a suitable (planar) Marangoni number can be defined. We have

$$
M=-4 \sigma_{0} k_{a} d^{2} \alpha_{3} \beta / k_{\|} \rho \eta_{a} k_{1} .
$$

Thus the b.c. at the open interface becomes

$$
D^{2} w=-a^{2} M \theta \text { at } Z=1
$$

as already indicated in section 2 .
The following results have been found :

$$
M_{\mathrm{c}}=-17.59, \quad a_{\mathrm{c}}=2.6
$$

correspond to the onset of steady convection. For a one-millimeter layer thickness it yields

$$
\Delta T_{\mathrm{c}}=-0.165^{\circ} \mathrm{C}
$$

(heating from below). Further, the possibility of destabilization of the layer by heating it from above has also been found. This case corresponds to

$$
M_{\mathrm{c}}=511.6, \quad a_{\mathrm{c}}=0.5 \text {, }
$$

which for a one-millimeter layer thickness corresponds to $\Delta T_{\mathrm{c}}=4.8^{\circ} \mathrm{C}$ (heating from above) for the onset of steady convection.

3.4.2 Homeotropic configuration. - In this case the b.c. lead a (homeotropic) Marangoni number

$$
M=-4 \sigma_{0} k_{a} \alpha_{2} d^{2} \beta /\left(k_{\|}+k_{a}\right) \eta_{b} k_{3}
$$

and the following results have been found :

(i) Steady transitions: $M_{\mathrm{c}}=40.6, a_{\mathrm{c}}=2.15$, which for a one-millimeter layer thickness yields

$$
\Delta T_{\mathrm{c}}=0.048^{\circ} \mathrm{C}
$$

(heating from above).

(ii) Overstable modes appear at $M_{\mathrm{c}}=-0.32 \times 10^{5}$ (heating from below) with $a_{\mathrm{c}}=2.42$, which for a one-millimeter layer thickness yields $\Delta T_{\mathrm{c}}=-37.7^{\circ} \mathrm{C}$. The associated period of oscillation is 119 seconds.

Lastly for both configurations the following general relation comes in a straightforward way from the equations

$$
\frac{R}{R_{\mathrm{c}}(M=0)}+\frac{M}{M_{\mathrm{c}}(R=0)} \approx 1
$$

as already obtained in a heuristic way by Guyon and Velarde [5].

Acknowledgments. - The authors wish to express their thanks to E. Guyon, J. Salan and E. DuboisViolette for many fruitful discussions. They are particularly grateful to E. Guyon, P. Pieranski and J. Salan for communicating to them several experimental findings prior to their publication. J. Salan has taken the pain of helping the authors with notation problems and a thorough revision of the manuscript. Remarks from two anonymous referees are also acknowledged. This research has been sponsored by the Instituto de Estudios Nucleares (Spain). 


\section{References}

[1] Dubois-Violette, E., C. R. Hebd. Séan. Acad. Sci. B 273 (1971) 923. See also Solid State Commun. 14 (1974) 767

[2] CURrie, P. K., Rheol. Acta 12 (1973) 165.

[3] Barratt, P. J. and Sloan, D. M., J. Phys. A 9 (1976) 1987.

[4] LeK Kerkerker, H. N. W., J. Physique Lett. 38 (1977) L-277.

[5] Guyon, E. and Velarde, M. G., J. Physique Lett. 39 (1978) L-205.

[6] Normand, Ch., Pomeau, Y. and Velarde, M. G., Rev. Mod. Phys. 49 (1977) 581.

[7] Schechter, R. S., Velarde, M. G. and Platten, J. K., $A d v$. Chem. Phys. 26 (1974) 265.

[8] Guyon, E. and Pieranski, P., C. R. Hebd. Séan. Acad. Sci. B 274 (1972) 656.

[9] Pieranski, P., Dubois-Violette, E. and Guyon, E., Phys. Rev. Lett. 30 (1973) 736.
[10] Salan, J., Guyon, E. and Pieranski, P., Procs. Intern. Symposium Liquid Crystals (Bordeaux, 1978)

[11] Guyon, E., Pieranski, P. and Salan, J., C. R. Hebd. Sium. Acad. Sci. Paris 287B (1978) 41.

[12] Guyon, E., Pieranski, P. and Salan, J., J. Fluid Mech. (to appear).

[13] Finlayson, B. A., The Method of weighted residuals and variational principles (Academic Press, New York) 1972, Chap. 6.

[14] DE Gennes, P. G., The physics of liquid crystals (Clarendon Press, Oxford) 1974.

[15] Chandrasekhar, S., Liquid crystals (Cambridge Univ. Press, Cambridge) 1977.

[16] Saupe, A., Mol. Cryst. Liq. Cryst. 7 (1969) 59. 